The following paper posted here is not the official IEEE published version. The final published version of this paper can be found in the Proceedings of the IEEE International Conference on Acoustics, Speech and Signal Processing (2007 : Honolulu, Hawaii):pp.II-865-II-868

Copyright (c) 2007 IEEE.

Personal use of this material is permitted. However, permission to reprint/republish this material for advertising or promotional purposes or for creating new collective works for resale or redistribution to servers or lists, or to reuse any copyrighted component of this work in other works must be obtained from the IEEE. 


\section{INTERVAL-BASED MAXIMUM LIKELIHOOD BENCHMARK FOR ADAPTIVE SECOND-ORDER ASYNCHRONOUS CCI CANCELLATION}

\author{
Alexandr M. Kuzminskiy* \\ Bell Laboratories \\ Alcatel-Lucent \\ The Quadrant, Swindon SN5 7DJ, UK \\ E-mail: ak9@alcatel-lucent.com
}

\author{
Yuri I. Abramovich
}

\author{
Intelligence, Surveillance \& \\ Reconnaissance Division, DSTO \\ PO Box 1500, Edinburgh SA 5111, Australia \\ Email: Yuri.Abramovich@dsto.defence.gov.au
}

\begin{abstract}
A potential usefulness of a priori time-of-arrival (TOA) information for asynchronous co-channel interference (CCI) cancellation is addressed. An interval-based maximum likelihood (ML) benchmark is developed and compared to the averaged ML benchmark and regularized second-order semi-blind (SB) solution that do not use the TOA information. It is found that in short burst scenarios the SB algorithm demonstrates performance that is close to the interval-based ML benchmark. Furthermore, it is shown that SB may outperform the ML benchmarks in the ML breakdown situation. For longer bursts, the known TOA information can significantly improve the performance.
\end{abstract}

Index Terms- Asynchronous co-channel interference, semiblind second-order filtering, interference time-of-arrival, maximum likelihood benchmark.

\section{INTRODUCTION}

Space-time (frequency) equalization and interference cancellation techniques in wireless communications usually exploit known training (pilot) symbols to estimate the weights of an antenna array. The underlying assumption for these techniques is that the training data is reliable since the co-channel interference (CCI) overlaps with the training symbols of the desired signal. Normally, this is the case for the synchronous CCI, which has the same time-frequency structure as the desired user. Asynchronous cells, packed transmission, interference avoidance schemes, and other techniques lead to more complicated asynchronous or intermittent CCI scenarios [1] - [3] and others, where the interference may partially overlap or not overlap with the training data of the desired signal. For example, a cross-layer analysis in [2] demonstrated that this kind of interference is especially relevant for visiting (distant) users in an Open Access Network (OAN) built on wireless local area network (WLAN) with medium access control (MAC) based on carrier sensing multiple access with collision avoidance (CSMA/CA).

A second-order statistic adaptive SB algorithm for asynchronous CCI cancellation is proposed and studied in [4], [5]. It is based on regularization of the conventional training-based least squares (LS) solution by means of the weighted covariance matrix estimated over

* Part of this work has been done in the context of the IST FP6 MEMBRANE project. the whole data interval. It is shown in [4], [5] that its performance in typical asynchronous CCI scenarios is close to the performance of a non-asymptotic ML benchmark jointly estimated over both the training and working intervals. This benchmark is based on the stochastic ML bounds developed in [6].

Similarly to the SB algorithm, the benchmark in [4], [5] uses the averaged estimates of the covariance matrices over the training and working intervals without recovering a temporal interference structure, i.e., TOA for different interference components. Potentially, TOA could be obtained by means of some detection of abrupt changes algorithms [7]. It is clear that this additional a priori information could be taken into account by interference cancellation algorithms. Obviously, in some scenarios the additional TOA information may be critically important. For example, if non-overlapping intervals affected by different interference components could be found at the data interval, then these intervals could be processed using different weight vectors instead of one set of weights trying to cancel all the interference components as in the average SB solution in [4], [5]. In other scenarios an advantage of such modified algorithm may be less obvious.

Taking into account that TOA estimation may be a difficult problem especially for short intervals, it is important to develop a modified interval-based non-asymptotic ML benchmark assuming the known TOA information. This benchmark can be useful for assessment of the potential performance in particular scenarios. If the interval-based ML benchmark demonstrates close to the averaged ML benchmark or SB performance, then additional efforts for TOA obtaining may not be justified in such scenarios.

In this paper we develop such an interval-based non-asymptotic benchmark and apply it in the particular asynchronous CCI scenario typical for visiting user collisions in an OAN WLAN system with CSMA/CA [2]. We show that for short data bursts the interval-based and averaged benchmarks demonstrate close performance. Furthermore, in the high signal-to-noise (SNR) case the SB algorithm may outperform the benchmarks because of the ML breakdown. On the contrary, for relatively long bursts with the low probability to meet short intervals with different interference scenarios, the additional TOA information may significantly increase the potential performance.

In Section 2 we describe the data model and formulate the problem. In Section 3 an interval-based non-asymptotic ML benchmark is developed. The simulation results are given in Section 4. Section 5 concludes the paper. 


\section{DATA MODEL AND PROBLEM FORMULATION}

We consider the following narrowband, e.g., for a separate OFDM sub-carrier, data model of the signal received by an antenna array of $K$ elements:

$$
\mathbf{x}(n)=\mathbf{h} s(n)+\sum_{m=1}^{M} \mathbf{g}_{m} u_{m}(n)+\mathbf{z}(n),
$$

where $n=1 \ldots N$ is the time index; $\mathbf{x}(n) \in \mathcal{C}^{K \times 1}$ is the vector of observed outputs of an antenna array; $s(n)$ is the desired signal, $\mathrm{E}\left\{|s|^{2}\right\}=p_{s}, \mathrm{E}\left\{s(q) s^{*}(g)\right\}=0, q \neq g, \mathrm{E}\{\cdot\}$ denotes expectation; $u_{m}(n), m=1 \ldots M$ are the $M<K-1$ components of CCI:

$$
\mathrm{E}\left\{u_{m}(q) u_{m}^{*}(g)\right\}=\left\{\begin{array}{ll}
p_{m}, & q=g \in \mathcal{N}_{m} \\
0, & q=g \ni \mathcal{N}_{m} \\
0, & q \neq g
\end{array},\right.
$$

$\mathcal{N}_{m}$ is the appearance interval for the $m$-th interference component, $\mathbf{z}(n) \in \mathcal{C}^{K \times 1}$ is the vector of noise, $\mathrm{E}\left\{\mathbf{z}(n) \mathbf{z}^{*}(n)\right\}=p_{0} \mathbf{I}_{K}$, $\mathrm{E}\left\{\mathbf{z}(q) \mathbf{z}^{*}(g)\right\}=0, q \neq g$ and $\mathbf{h} \in \mathcal{C}^{K \times 1}$ and $\mathbf{g}_{m} \in \mathcal{C}^{K \times 1}$ are the vectors modelling linear propagation channels for the desired signal and interference. All propagation channels are assumed to be stationary over the whole data slot and independent for different antenna elements and slots. The desired signal, noise, and all interference components are assumed to be independent circular Gaussian processes.

The training interval of $N_{\mathrm{t}}>K+1$ samples: $s(n), n=$ $1, \ldots, N_{\mathrm{t}}$, is locaded in the preamble and known at the receiver. The working data interval $n=N_{\mathrm{t}}+1, \ldots, N$ of $N_{\mathrm{d}}=N-N_{\mathrm{t}}$ samples is defined as the rest of the slot.

The interference appearance intervals $\mathcal{N}_{m}=n_{m}, \ldots, N$ of $N_{m}=N-n_{m}+1$ samples, where $n_{m+1}>n_{m}>N_{t}$ for $m=1, \ldots, M-1$, are assumed to be known at the receiver for the interval-based ML benchmark and not known for the SB algorithm and averaged ML benchmark. All the propagation channels, interference and noise power are not known at the receiver.

The introduced model is illustrated in Fig. 1. It corresponds to the situation where the CCI components in a "hidden terminal" environment appear with random delays because of the CSMA/CA protocol as shown in [2]. The important feature of this model is that some of the intervals with different interference scenarios in Fig. 1 may be quite short leading to estimation difficulties.

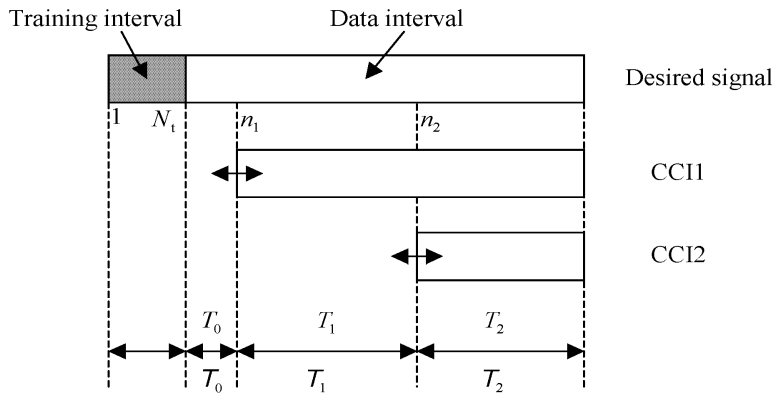

Fig. 1. Narrowband data model for two-component asynchronous CCI

Similarly to [4], [5], for simplification of Gaussian modelling we assume that the training samples are generated as i.i.d. complex Gaussian random values. While the actual power of the useful signal $p_{s}$ is unknown, the power of the training signal $p_{\mathrm{t}}$ is set to 1. Note, that this modelling means that strictly speaking we have to simulate different random-like training sequences to accurately fit into the Gaussian assumption. In what follows for analytical derivations we accept Gaussian random-like training signal model, while for actual simulations we model the desired signal and CCI as independent streams of random $( \pm 1 \pm j 1) / \sqrt{2}$ symbols. Another simplification is that we assume that all the intervals $\mathcal{T}_{m}$ of $T_{m}$ symbols, $m=0, \ldots, M$ with different interference scenarios are long enough to form sufficcient statatistics for each interval, i.e., $T_{m}>K$. This assumption significantly simplifies Gaussian modelling and do not change the overall results significantly.

A signal estimate can be found as the output of a spatial filter:

$$
\hat{s}(n)=\hat{\mathbf{w}}^{*}(n) \mathbf{x}(n), n=N_{\mathrm{t}}+1, \ldots, N .
$$

In the case of averaged or "stationarized" processing a fixed weight vector $\hat{\mathbf{w}}(n)=\hat{\mathbf{w}}$ is used for the whole data interval. Particularly, the SB solution [4], [5] can be expressed as follows:

$$
\hat{\mathbf{w}}_{\mathrm{SB}}=\left[(1-\delta) \hat{\mathbf{R}}_{\mathrm{t}}+\delta \hat{\mathbf{R}}_{\mathrm{d}}\right]^{-1} \hat{\mathbf{r}}_{\mathrm{t}},
$$

where $\hat{\mathbf{R}}_{\mathrm{t}}=N_{\mathrm{t}}^{-1} \sum_{1}^{N_{\mathrm{t}}} \mathbf{x}(n) \mathbf{x}^{*}(n)$ and $\hat{\mathbf{r}}_{\mathrm{t}}=N_{\mathrm{t}}^{-1} \sum_{1}^{N_{\mathrm{t}}} s^{*}(n) \mathbf{x}^{*}(n)$ are the covariance matrix and cross-correlation vector estimated over the training interval, $\hat{\mathbf{R}}_{\mathrm{d}}=N_{\mathrm{d}}^{-1} \sum_{n=N_{\mathrm{t}^{+1}}}^{N} \mathbf{x}(n) \mathbf{x}^{*}(n)$ is the covariance matrix estimated over the whole data interval and $0 \leq \delta \leq$ 1 is the regularization coefficient.

One can see that the SB estimator (4) contains the conventional LS solution $\hat{\mathbf{w}}_{\mathrm{LS}}=\hat{\mathbf{R}}_{\mathrm{t}}^{-1} \hat{\mathbf{r}}_{\mathrm{t}}$ as a particular case for $\delta=0$.

In the case of an interval-based processing, it is naturally to apply different spatial filters at different intervals $\mathcal{T}_{m}$ of $T_{m}$ symbols:

$$
\hat{\mathbf{w}}(n)=\hat{\mathbf{w}}_{m}, n \in \mathcal{T}_{m}, m=0, \ldots, M .
$$

The problem is to find a ML bound for such estimates and compare its efficiency with the average ML benchmark [4], [5] and the SB algorithm (4) that do not use the TOA information.

\section{INTERVAL-BASED NON-ASYMPTOTIC ML BENCHMARK}

\subsection{Optimization criterion}

Under the introduced data model ${ }^{1}$, the covariance matrices at the training and data intervals are:

$$
\begin{gathered}
\mathbf{R}_{\mathrm{t}}=\mathbf{v v}^{*}+p_{0} \mathbf{I}_{K}, \\
\mathbf{R}_{m}=\mathbf{R}_{m-1}+\mathbf{g}_{m} \mathbf{g}_{m}^{*}, m=1, \ldots, M,
\end{gathered}
$$

where $\mathbf{R}_{0}=\mathbf{R}_{\mathbf{t}}$ and $\mathbf{v}=\sqrt{p_{s}} \mathbf{h}$.

During the training interval we observe $(K+1)$-dimension independent Gaussian training vectors $\overline{\mathbf{x}}(n)=\left[s(n), \mathbf{x}^{T}(n)\right]^{T}, n \in \mathcal{N}_{\mathrm{t}}$. Taking into account that $N_{\mathrm{t}}>K+1$, the sufficient statistic at the training interval is

$$
\hat{\overline{\mathbf{R}}}_{\mathrm{t}}=\mathbf{N}_{\mathrm{t}}^{-1} \sum_{\mathbf{n}=\mathbf{1}}^{\mathbf{N}_{\mathrm{t}}} \overline{\mathbf{x}}(\mathbf{n}) \overline{\mathbf{x}}^{*}(\mathbf{n})=\left[\begin{array}{cc}
\hat{p}_{\mathrm{t}} & \hat{\mathbf{r}}_{\mathrm{t}}^{*} \\
\hat{\mathbf{r}}_{\mathrm{t}} & \hat{\mathbf{R}}_{\mathrm{t}}
\end{array}\right],
$$

\footnotetext{
${ }^{1}$ Some simplifications are adopted for the data model in Section 2 such as no $\mathrm{CCI}$ at the training interval, $T_{m}>K$ and all the CCI components last till the end of the data burst. The proposed benchmark can be easily modified for the general scenario, e.g., undersumpled case $T_{m}<K$ can be addressed similarly to $[8]$
} 
where $\hat{\mathbf{p}}_{\mathrm{t}}=N_{\mathrm{t}}^{-1} \sum_{n=1}^{N_{\mathrm{t}}} s(n)^{*} s(n), \hat{\mathbf{r}}_{\mathrm{t}}=N_{\mathrm{t}}^{-1} \sum_{n=1}^{N_{\mathrm{t}}} s(n)^{*} \mathbf{x}(n)$, $\hat{\mathbf{R}}_{\mathrm{t}}=N_{\mathrm{t}}^{-1} \sum_{n=1}^{N_{\mathrm{t}}} \mathbf{x}(n) \mathbf{x}^{*}(n)$ and

$$
\mathrm{E}\left\{\hat{\mathbf{R}}_{\mathrm{t}}\right\}=\left[\begin{array}{ll}
1 & \mathbf{v}^{*} \\
\mathbf{v} & \mathbf{R}_{\mathrm{t}}
\end{array}\right] .
$$

During the working interval we observe $K$-dimension independent Gaussian vectors $\mathbf{x}(n), n \in \mathcal{T}_{m}$ and again because of $T_{m}>K$, the sufficient statistics at the working intervals are $\hat{\mathbf{R}}_{m}=$ $T_{m}^{-1} \sum_{n \in \mathcal{T}_{m}} \mathbf{x}(n) \mathbf{x}^{*}(n)$, where $\mathrm{E}\left\{\hat{\mathbf{R}}_{m}\right\}=\mathbf{R}_{m}$ for $m=0, \ldots, M$.

Taking into account the structure of covariance matrices at different intervals, the admissible set of the optimization parameters can be introduced as follows:

$$
\begin{gathered}
\overline{\mathbf{A}}_{\mathbf{t}}=\left[\begin{array}{ll}
1 & \mathbf{b}^{*} \\
\mathbf{b} & \mathbf{b b}^{*}+d \mathbf{I}_{K}
\end{array}\right]>0, \\
\mathbf{A}_{m}=\mathbf{A}_{m-1}+\mathbf{c}_{m} \mathbf{c}_{m}^{*}>0, m=1, \ldots, M, \\
\mathbf{A}_{0}=\mathbf{b b}^{*}+d \mathbf{I}_{K}>0,
\end{gathered}
$$

where $\mathbf{b}$ and $\mathbf{c}_{m}, m=1, \ldots, M$ are a $K \times 1$ vectors and $d$ is a positive scalar.

Since sufficient statistics $\hat{\overline{\mathbf{R}}}_{\mathrm{t}}$ and $\hat{\mathbf{R}}_{m}$ are non-degenerate matrices, the ML estimates could be obtained via maximization of a monotonic function of the product of the $M+2$ likelihood ratios (LR):

$$
\begin{gathered}
\text { Find } \max _{\mathbf{b}, \mathbf{c}_{m}, d} \gamma\left(\overline{\mathbf{A}}_{\mathbf{t}}, \mathbf{A}_{m}\right) \\
\gamma\left(\overline{\mathbf{A}}_{\mathbf{t}}, \mathbf{A}_{m}\right)=\gamma_{\mathrm{t}}\left(\overline{\mathbf{A}}_{\mathrm{t}}\right) \prod_{m=0}^{M} \gamma_{m}\left(\mathbf{A}_{m}\right)^{T_{m} / N_{\mathrm{t}}}, \\
\gamma_{\mathbf{t}}\left(\tilde{\mathbf{A}}_{\mathbf{t}}\right)=\frac{\operatorname{det}\left(\overline{\mathbf{A}}_{\mathbf{t}}^{-1} \hat{\mathbf{R}}_{\mathrm{t}}\right) \exp (K+1)}{\exp \left[\operatorname{tr}\left(\tilde{\mathbf{A}}_{\mathbf{t}}^{-1} \hat{\tilde{\mathbf{R}}}_{\mathrm{t}}\right)\right]} \\
\gamma_{m}\left(\mathbf{A}_{m}\right)=\frac{\operatorname{det}\left(\mathbf{A}_{m}^{-1} \hat{\mathbf{R}}_{m}\right) \exp (K)}{\exp \left[\operatorname{tr}\left(\mathbf{A}_{m}^{-1} \hat{\mathbf{R}}_{m}\right)\right]}
\end{gathered}
$$

\subsection{Initialization}

For Monte-Carlo simulations in Section 4 we adopt an approximate recursive procedure that estimates vector $\mathbf{c}_{m}$ assuming that matrix $\mathbf{A}_{m-1}$ is known:

$$
\begin{gathered}
d^{[0]}=(K-1)^{-1} \sum_{i=1}^{K-1} \alpha_{i}, \mathbf{b}^{[0]}=\mathbf{c}_{0}^{[0]}=\hat{\mathbf{r}}_{\mathrm{t}}, \\
\mathbf{c}_{m}^{[0]}=\sqrt{\lambda_{\max }} \hat{\mathbf{A}}_{m-1}^{-\frac{1}{2}} \mathbf{v}_{\max }, \\
\hat{\mathbf{A}}_{m}=\hat{\mathbf{A}}_{m-1}+\hat{\mathbf{c}}_{m}^{[0]} \hat{\mathbf{c}}_{m}^{[0] *}, m=1, \ldots, M,
\end{gathered}
$$

where $\lambda_{\max }$ and $\mathbf{v}_{\max }$ are the maximum eigenvalue and the corresponding eigenvector of matrix $\hat{\mathbf{A}}_{m-1}^{-\frac{1}{2}} \hat{\mathbf{R}}_{m} \hat{\mathbf{A}}_{m-1}^{-\frac{1}{2}}$ and $\alpha_{i}$ are the eigenvalues of matrix $\hat{\mathbf{R}}_{\mathrm{t}}$ in ascending order.

The ML estimate of a partially known covariance matrix used in (18), (19) is based on the algorithm developed in [9]. Other initialization procedures, e.g., similar to [4], [5], can be applied as well.

\subsection{Outliers selection}

As far as the optimization problem formulated in Section 3.1 is not convex, any initialization and locally convergent optimization algorithm cannot guarantee finding the global solution. A technique that allows us to decide if the local solution is far away from the global maximum, i.e. it can be classified as an outlier, is developed in [6]. For benchmarking applications as in this paper, the outliers can be just disregarded and performance can be estimated over remaining trials [4], [5].

The basic idea is that the global maximum of the LR function (13) always exceeds the LR function for the actual parameters, i.e. for the global solutions $\left(\hat{\mathbf{R}}_{\mathrm{t}}\right)_{\mathrm{ML}}$ and $\left(\hat{\mathbf{R}}_{m}\right)_{\mathrm{ML}}$ we have

$$
\left.\gamma_{\max }=\gamma\left[\left(\hat{\mathbf{R}}_{\mathrm{t}}\right)_{\mathbf{M L}},\left(\hat{\mathbf{R}}_{\mathbf{m}}\right)_{\mathrm{ML}}\right]>\gamma\left(\overline{\mathbf{R}}_{\mathrm{t}}, \mathbf{R}_{m}\right)\right)=\gamma_{0} .
$$

In this paper we apply the interval-based ML benchmark only to simulated data. So, the selection procedure is as follows:

- Find a local solution to the nonlinear optimization problem (13) using the presented initialization and calculate the LR value for this solution $\hat{\gamma}=\gamma\left(\hat{\mathbf{R}}_{\mathbf{t}}, \hat{\mathbf{R}}_{\mathbf{m}}\right)$.

- If $\hat{\gamma}>\gamma_{0}$, accept this solution, otherwise classify it as an outlier.

\section{SIMULATION RESULTS}

We simulate a four-element antenna array and two-component interference according to the asynchronous CCI scenario in Fig.1. The desired signal and interference are generated as independent streams of random symbols $( \pm 1 \pm 1) / \sqrt{2}$. All propagation channels are simulated as independent complex Gaussian vectors with unit variance and zero mean. The training sequence of $N_{\mathrm{t}}=8$ symbols and variable total number of symbols $N$ are considered. The interval-based ML benchmark presented in Section 3, the averaged ML benchmark from [4], [5] and the SB algorithm (4) are compared by means of the mean square error (MSE) and bit error rate (BER) performance estimated over $10^{4}$ trials with independent channel and TOA realizations.

The MSE and BER results are presented in Fig. 2, 3 for variable SNR and different burst duration. The benchmark performance is estimated over (70 - 95)\% of trials depending on conditions using the outlier selection procedure specified in Section 3.3. First of all, one can see that for all considered scenarios the LS performance is very poor compared to SB and both benchmarks as expected. Furthermore, for shorter bursts the interval-based ML advantage over the average benchmark and SB is not significant: it is less than $2 \mathrm{~dB}$ for low and medium SNR in Fig. 2. For longer bursts, the interval-based ML benchmark shows much better results, e.g., $4 \mathrm{~dB}$ and $6 \mathrm{~dB}$ improvement at $0.1 \%$ BER compared to the averaged ML benchmark and $\mathrm{SB}$ as in Fig. 3.

An important observation can be made for the high SNR in Fig. 2, where the SB algorithm outperforms both the ML benchmarks. A possible reason is the ML breakdown for short overlapping intervals, where the ML solution becomes unreliable and leads to poor estimates in terms of MSE and BER. Obviously, short overlapping intervals can be met with higher probability in a short burst scenario and their average impact is more significant for higher SNR. This is why the ML performance degradation in our simulations is visible only in Fig. 2 for $\mathrm{SNR}=20 \mathrm{~dB}$. 
This effect is further illustrated in Fig. 4 that presents the MSE and BER performance in the scenario shown in Fig. 1 with $n_{1}=$ $n_{2}=N_{\mathrm{t}}+1$, i.e., both interference components completely overlap with the data interval. The fixed size of the training interval of $N_{\mathrm{t}}=$ 8 symbols and variable duration of the data interval are assumed in Fig. 4. Clearly, both the ML benchmarks are equivalent in this case. Again, one can see that for very short data interval of $N_{\mathrm{d}}=5$ symbols, SB outperforms the ML benchmark; for medium size data interval of $N_{\mathrm{d}}=10$ symbols, their performance is almost the same; and for longer data interval of $N_{\mathrm{d}}=42$ symbols, the ML benchmark clearly outperforms the SB solution. It is worth emphasizing that in all these scenarios the SB solution is very poor in terms of ML estimation. For example, in the case of $N_{\mathrm{d}}=5, \hat{\gamma}_{\mathrm{SB}} / \gamma_{0}<0.1$ is observed in more that $90 \%$ of trials for $\mathrm{SNR}=15 \mathrm{~dB}$.

\section{CONCLUSION}

An asynchronous interference cancellation problem has been considered. A potential usefulness of an a priori TOA information has been studied. An interval-based ML benchmark has been developed and compared to the averaged ML benchmark and SB solution that do not use the TOA information. It has been found that in short burst scenarios the SB algorithm demonstrates performance that is close to the interval-based ML benchmark. Furthermore, it may outperform the ML benchmarks in the ML breakdown situation. For longer bursts, the known TOA information can significantly improve the performance. Thus, new second-order semi-blind algorithms with TOA estimation may be required for such scenarios.

\section{REFERENCES}

[1] J. Karlsson, J. Heinegard, "Interference rejection combining for GSM," in Proc. ICUPC, pp.433-437, 1996.

[2] A. M. Kuzminskiy, H. R. Karimi "Cross-layer design of uplink multiple-antenna interference cancellation for WLAN with CSMA/CA in open acess networks," to appear in ICC, 2007.

[3] M. Nicoli, M. Sala, O. Simeone, L. Sampietro, C. Santacesaria, "Adaptive array processing for time-varing interference mitigation in IEEE 802.16 systems," in Proc. PIMRC, Helsinki, 2006.

[4] A. M. Kuzminskiy, Y. I. Abramovich, "Adaptive second-order asynchronous CCI cancellation: maximum likelihood benchmark for regularized semi-blind technique," in Proc. ICASSP, vol. 4, pp. 453-456, Montreal, 2004.

[5] A. M. Kuzminskiy, Y. I. Abramovich, "Second-order asynchronous interference cancellation: Regularized semi-blind technique and non-asymptotic maximum likelihood benchmark," Signal Processing, vol. 86, no. 12, pp. 3849-3863, 2006.

[6] Y. I. Abramovich, N. K. Spencer, A. Y. Gorokhov, "Bounds on maximum likelihood ratio-part I: application to antenna array detection-estimation with perfect wavefront coherence," IEEE Trans. Signal Processing, vol. 52, no. 6, pp. 1524 - 1536, 2004.

[7] M. Basseville, I. V. Nikiforov, "Detection of abrupt changes. Theory and applications," Prentice Hall, 1993.

[8] B. Johnson, Y. I. Abramovich, "GLRT-based detectionestimation of Gaussian signals in under-sampled training conditions," in Proc. EUSIPCO, Florence, 2006

[9] A. Medles, "Coding and advanced signal processing for MIMO systems," PhD Thesis, Telecom Paris (ENST) Institut Eurecom, 2004.
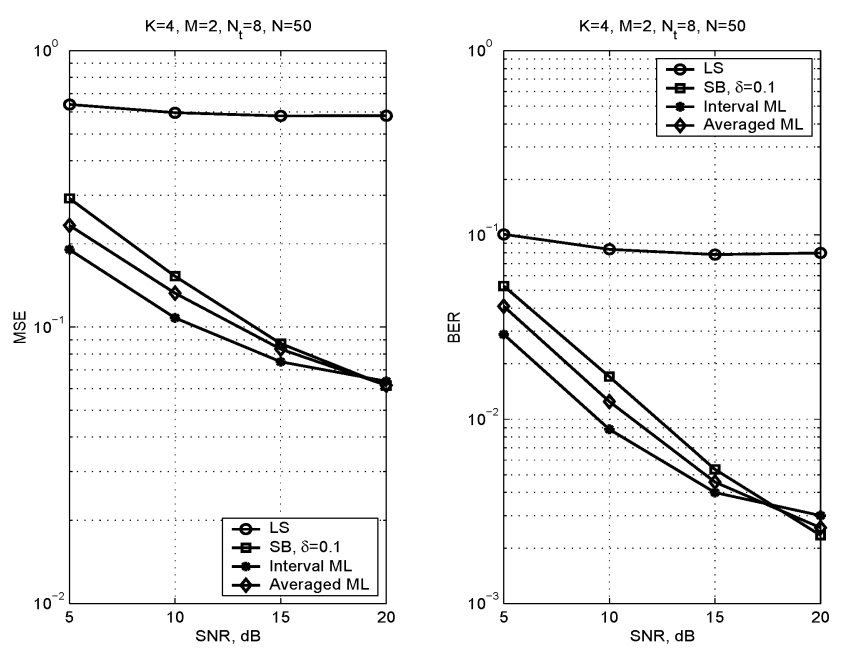

Fig. 2. MSE and BER performance for $N=50$
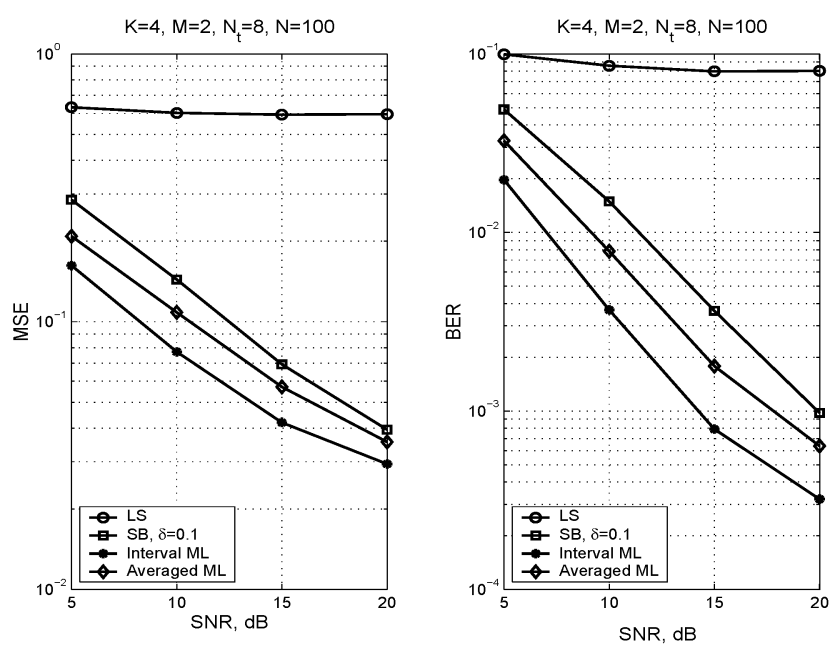

Fig. 3. MSE and BER performance for $N=100$
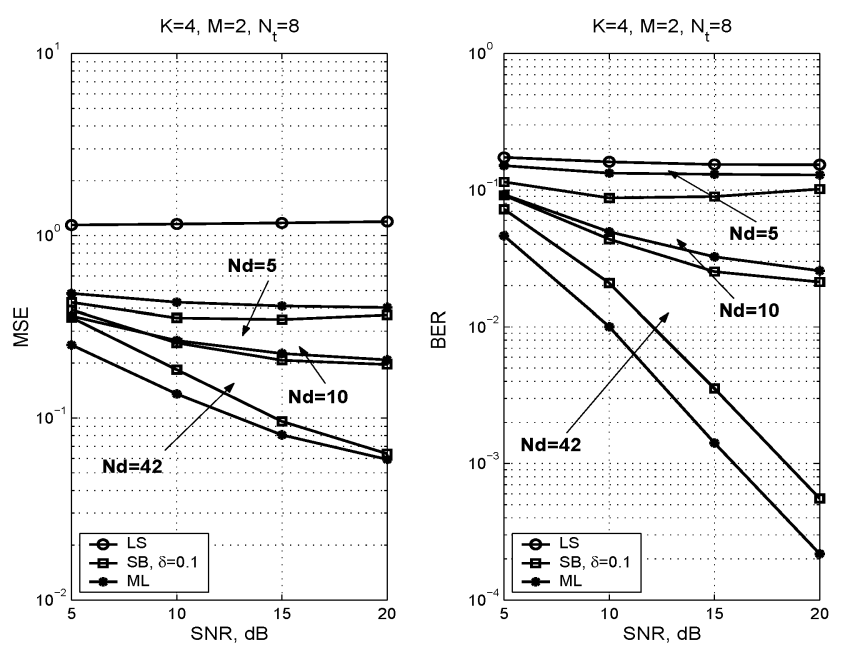

Fig. 4. Illustration of the ML breakdown 